Research Paper

\title{
Transient Effect of $17 \beta$-estradiol on Osteoporosis in Ovariectomized Rats Accompanied with Unilateral Disuse in the Early Phase
}

\author{
Xiaodi Sun ${ }^{1,3}$, Jin Liang1,3, Chune Wang2, Sensen $\mathrm{Cao}^{2}$, Yingwei $\mathrm{Hu}^{2}{ }^{\circledR}$ and $\mathrm{Xin}^{\mathrm{X}} \mathrm{u}^{1,3}$ \\ 1. School of Stomatology, Shandong University, Wenhuaxi Road 44-1, Jinan 250012, China. \\ 2. Institute of Dental Medicine, Qilu Hospital, Shandong University, Wenhuaxi Road 107, Jinan 250012, China. \\ 3. Shandong Provincial Key Laboratory of Oral Biomedicine, Jinan, China.
}

$\triangle$ Corresponding authors: Xin Xu, School of Stomatology, Shandong University, Wenhuaxi Road 44-1, Jinan 250012, China. Telephone: +86531 88382595, Email: xinxu@sdu.edu.cn, Fax: +86 531 88382923. Yingwei Hu, Institute of Dental Medicine, Qilu Hospital, Shandong University, Wenhuaxi Road 107, Jinan 250012, China. Telephone: + 86531 82169286, Email: huyingwei@sdu.edu.cn, Fax: +86 53182169286.

(C) 2015 Ivyspring International Publisher. Reproduction is permitted for personal, noncommercial use, provided that the article is in whole, unmodified, and properly cited. See http://ivyspring.com/terms for terms and conditions.

Received: 2015.02.13; Accepted: 2015.05.13; Published: 2015.05.23

\begin{abstract}
Purpose: It is clinically important to determine the efficacy of estrogen replacement for postmenopausal women combined with mobility difficulties, due to the potential risks of estradiol. The objective of the current study was to investigate the effect of estradiol replacement on osteoporosis induced by the ovariectomy (OVX) combined with unilateral sciatic neurectomy (SN) in a rat model. Method: Female Sprague-Dawley rats were subjected to OVX and unilateral SN on the right hindlimb $(\mathrm{OVX}+\mathrm{SN})$ or sham surgery $(C T R L)$. 17 $\beta$-estradiol $\left(\mathrm{E}_{2}\right)$ or vehicle was administrated to the rats immediately, and followed by every other day. Bone mass and trabecular microarchitecture were analyzed using micro-Computed Tomography (micro-CT) and histology at days $3,7,14$, and 28 post-surgery. The local expressions of sclerostin/SOST, secreted exclusively by osteocytes, and tartrate-resistant acid phosphatase 5b (TRAP 5b), produced mostly by osteoclasts, were examined by immunohistochemistry and TRAP staining, respectively. Serum markers of bone resorption, including C-terminal telopeptides of type I collagen (CTx), receptor activator for nuclear factor $K B$ ligand (RANKL), and TRAP $5 b$, were quantified by enzyme linked immunosorbent assay (ELISA). Result: Based on micro-CT analysis, $\mathrm{E}_{2}$ treatment of $\mathrm{OVX}+\mathrm{SN}$ rats improved the preservation of the bone volume fraction (BV/TV) and trabecular number (Tb.N) in the tibias at day 14 post-surgery, which were $43 \%$ and $46 \%$ higher in $\mathrm{OVX}+\mathrm{SN}+\mathrm{E}_{2}$ rats than those in OVX+SN rats, respectively. However, the impact of $E_{2}$ was transient and disappeared at day 28. Expression of sclerostin in the tibias of $\mathrm{OVX}+\mathrm{SN}$ rats was significantly elevated at day 7 post-surgery compared with the CTRL, but was suppressed until day 14 with $E_{2}$ replacement. Conclusion: Our results showed that estrogen replacement could transiently protect against bone loss in OVX rats combined with mechanical unloading. The up-regulation of sclerostin expression appears to be transiently delayed by $E_{2}$ treatment in our models.
\end{abstract}

Key words: $E_{2}$ replacement; osteoporosis; estrogen deficiency; mechanical unloading; sclerostin

\section{Introduction}

Osteoporosis is a disease characterized by low bone mass and deterioration of bone tissue that can be caused by reduced estrogen levels and mechanical unloading $[1,2]$. Both estrogen and mechanical loading play essential roles in the maintenance of bone. It is widely recognized that estrogen loss at menopause or under various clinical conditions in younger women induces changes in bone turnover [3]. Similarly, musculoskeletal disuse caused by long-term immobilization and extended bed rest also results in a 
clinically significant bone loss.

Estrogen and mechanical loading also seem to exert synergistic effects on bone maintenance [4]. Disuse exacerbates the bone loss resulting from estrogen-deficiency [5], which presents a clinical concern to estrogen-deficient women experiencing extended bouts of disuse by stroke or other accidents. However, several meta-analyses reported that postmenopausal women experience very little, if any, elevation in bone volume after exercise intervention [6-10]. In contrast, exercise combined with estrogen replacement results in greater improvements in bone volume of postmenopausal women than either treatment alone [11, 12]. These observations suggest that the estrogen status significantly influences mechanical loading-induced bone proliferation. But it is still unclear whether the estrogen status has any effect on the bone turnover in the postmenopausal women combined with mechanical unloading.

Bone architecture is adjusted to be functionally appropriate for mechanical stimulation that initiates cascades of responses in resident bone cells which, in turn, influence the activity of bone turnover factors responsible for bone metabolism [13]. The activity of these cells and factors is also influenced by estrogens [14]. The combination of estrogen-deficiency and unilateral disuse with/without estrogen replacement might also aid to trigger bone remodeling and secretion of bone turnover markers. Some of the most potent markers for bone turnover include C-terminal telopeptides of type I collagen (CTx), receptor activator for nuclear factor $\mathrm{kB}$ ligand (RANKL), sclerostin/SOST, and the tartrate-resistant acid phosphatase $5 b$ (TRAP $5 b$ ).

Most previous studies focused on single intervening factor after the long-term menopause or disuse, and single time point. It is important to understand the developing process of bone turnover by estrogen deficiency or disuse in the early phase for effective clinical intervention. The current study aimed to investigate the effect of estrogen replacement on preventing osteoporosis by ovariectomy (OVX) with concomitant unilateral sciatic neurectomy (SN), and to elucidate the alterations of relevant bone turnover markers over a time course of 3, 7, 14, and 28 days in the early phase in a rat model. We hypothesized that estrogen treatment prevents, at least partly, the loss of bone in the OVX+SN bone.

\section{Materials and methods}

\section{Animals and experimental design}

Ninety-six 12-week-old female Sprague-Dawley rats were divided randomly into three groups $(\mathrm{n}=$ 32/group): OVX combined with unilateral SN, treated with or without $17 \beta$-estradiol $\left(\mathrm{E}_{2}\right)\left(\mathrm{OVX}+\mathrm{SN}+\mathrm{E}_{2}\right.$, $\mathrm{OVX}+\mathrm{SN}$, respectively), and sham operation as control (CTRL). The rats underwent trans-abdominal OVX or a sham operation under anesthesia with intraperitoneal ketamine hydrochloride $(100 \mathrm{mg} / \mathrm{kg}$ body weight). Unilateral SN was performed on the right hindlimb by resecting 4 to 5 -mm segment. After the operation, $\mathrm{OVX}+\mathrm{SN}+\mathrm{E}_{2}$ rats were injected with $\mathrm{E}_{2}$ at $10 \mu \mathrm{g} / \mathrm{kg}$ body weight (Sigma, St Louis, MO, USA) immediately and followed by every other day throughout the study. OVX+SN rats and CTRL rats were injected with vehicle. All experiments were conducted with the approval of the Shandong University Animal Care and Use Committee (Jinan, China).

Eight rats from each group were euthanized at days $3,7,14$, and 28 post-surgery, blood samples and the right tibias were collected. The tibias were fixed with $4 \%$ paraformaldehyde overnight. The serum was collected and kept in $-80^{\circ} \mathrm{C}$.

\section{Micro-CT analysis}

The proximal tibia was scanned using micro-CT (Skyscan 1172; Skyscan, Belgium) with an isotropic voxel size of $8 \mu \mathrm{m}$ at energy settings of $80 \mathrm{kV}$ and 80 $\mu \mathrm{A}$, using aluminum filter of $0.5 \mathrm{~mm}$. The images were reconstructed using NRecon software (Skyscan v. 1.6.8.0). Analyses of trabecular bone volume fraction (BV/TV, bone volume/tissue volume), bone surface density (BS/TV, bone surface/tissue volume), bone surface/volume ratio (BS/BV, bone surface/bone volume), and trabecular thickness, number, and separation (Tb.Th, Tb.N, and Tb.Sp, respectively) were performed in cancellous bone using CTAn (Skyscan v. 1.12.0.0). BV/TV was considered as a primary variable. Three-Dimension (3D) reconstructions were performed.

\section{Histological analysis}

After the micro-CT scanning, the proximal tibias were placed in $10 \%$ ethylene diamine tetraacetic acid (EDTA) for decalcification at $4{ }^{\circ} \mathrm{C}$ for about one month, followed by embedding and series section. Four- $\mu \mathrm{m}$ slices were sectioned on the coronal plane and stained with hematoxylin-eosin (H\&E). Images (10x) were taken using LEICA microscope (LEICA DM 4000 B) and imported into the analysis software (Image-Pro Plus 6.0) to calculate BV/TV in trabecula.

\section{ELISA analysis of serum markers}

Serum markers were quantified using commercial ELISA kits, RatTRAP for TRAP $5 b$ and RatLaps for CTx (Immunodiagnostic Systems, Gaithersburg, MD, USA), and RANKL ELISA Kit for RANKL (R\&D Systems, Minneapolis, MN, USA), following the 
manufacturer's instructions. All samples were assayed in duplicate.

\section{Immunohistochemistry}

Decalcified sections of the proximal tibia were probed with primary antibody against sclerostin (10 $\mu \mathrm{g} / \mathrm{ml}$; R\&D Systems) overnight at $4{ }^{\circ} \mathrm{C}$. Detection was achieved by using DAB kit (ZSGB-BIO, Beijing, China) followed by counterstaining with hematoxylin. The sections were photographed using $20 \times$ objective within cortical bone. The numbers of sclerostin-positive (sclerostin+) (stained brown) and -negative (sclerostin-) (stained blue) osteocytes were counted. The percentage of sclerostin-positive osteocytes out of the total number of osteocytes (sclerostin+ plus sclerostin-) was calculated.

\section{TRAP staining}

Four- $\mu \mathrm{m}$ slices of the proximal tibia were stained for TRAP 5b using Acid Phosphatase, Leukocyte (TRAP) Kit (Sigma) following the manufacturer's instruction. Within a distance of $0.2 \mathrm{~mm}$ from the curvature of the growth plate, 20 images were randomly collected using $40 \times$ objective under LEICA microscope (LEICA DM 4000 B). TRAP-positive cells

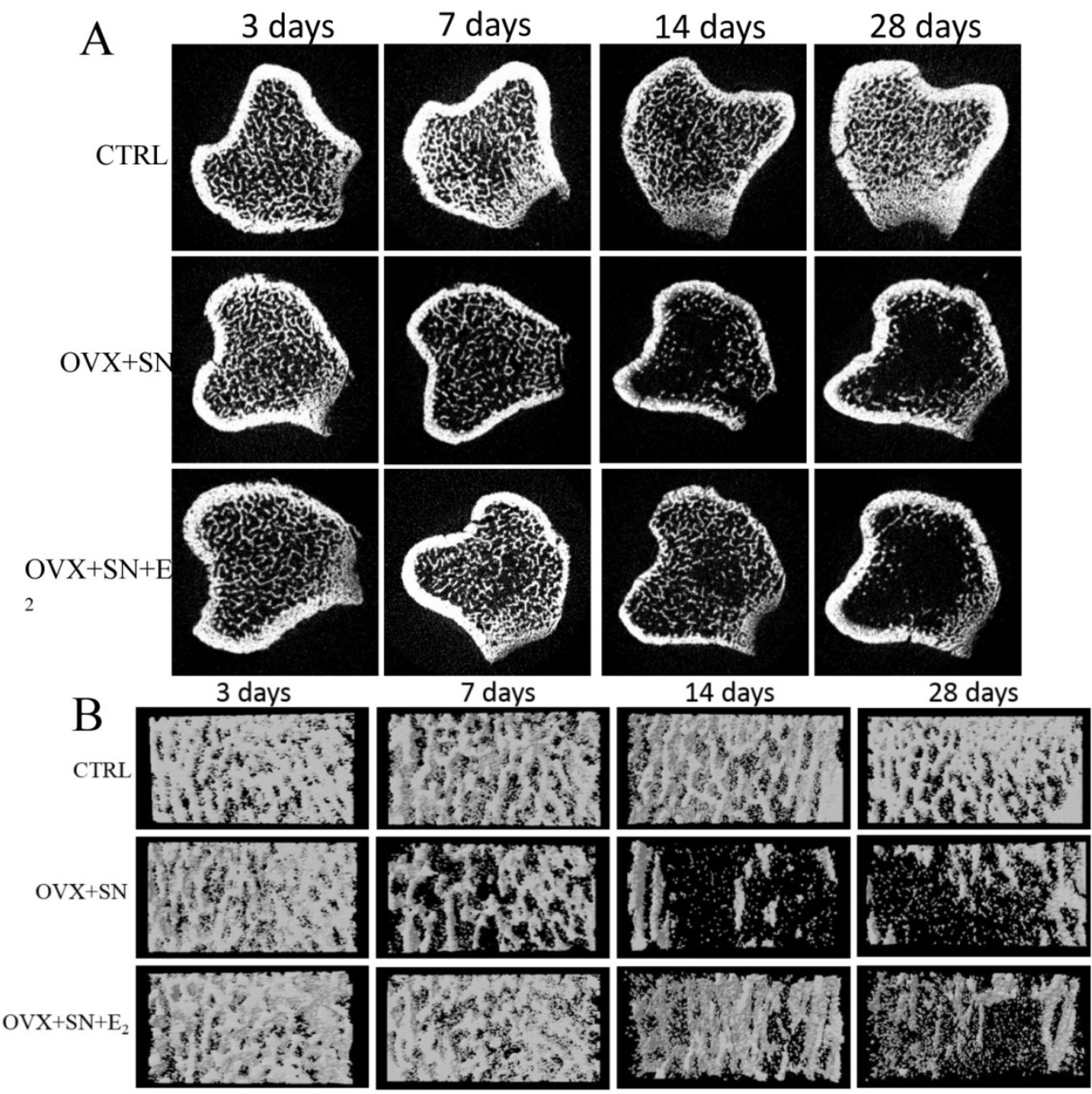

with more than three nuclei were counted as osteoclasts in the primary spongiosa of the proximal tibia $[15,16]$.

\section{Statistical analysis}

All data were expressed as the mean values \pm SEM. Differences between the experimental groups and control group were tested using one-way ANOVA and repeated separately for each time point examined. Analyses were performed using the GraphPad Prism 6.0 software program (GraphPad Software Inc., La Jolla, CA, USA). P-value less than 0.05 was considered statistically significant.

\section{Results}

\section{Transient effect of $E_{2}$ on osteoporosis induced by ovariectomy combined with sciatic neurectomy}

The representative cross-sectional images and 3-Dimension reconstruction of cancellous bone from micro-CT analysis in the proximal tibias were shown in Fig. $1 \mathrm{~A}$ and $1 \mathrm{~B}$, respectively. There was no change in the microstructure of trabecula over time in sham rats (Fig. 1A, B; CTRL). OVX+SN caused visible bone loss at day 7 and significant loss at days 14 and 28 (Fig. 1A, B; $\mathrm{OVX}+\mathrm{SN}) . \mathrm{E}_{2}$ treatment inhibited the bone loss until day 14; the bone loss was very minor at day 14 , but became significant at day 28 (Fig. $1 \mathrm{~A}, \mathrm{~B} ; \mathrm{OVX}+\mathrm{SN}+\mathrm{E}_{2}$ ).

Fig. I Representative micro-CT images of the proximal tibia in rats undergoing OVX+SN with/without $\mathrm{E}_{2}$ replacement and control. (A) Representative cross-sectional images show the ongoing changes of trabecular microarchitecture. At day 14 post-surgery, the cancellous bone in OVX+SN rats show more serious bone destruction than that in $\mathrm{OVX}+\mathrm{SN}+\mathrm{E}_{2}$ rats. At day 28 post-surgery, the cancellous bone in both $\mathrm{OVX}+\mathrm{SN}$ and $\mathrm{OVX}+\mathrm{SN}+\mathrm{E}_{2}$ rats were seriously deteriorated. (B) The representative three-dimensional images of the proximal tibia. The trabecular bone in $\mathrm{OVX}+\mathrm{SN}+\mathrm{E}_{2}$ rats is significantly more than that in $\mathrm{OVX}+\mathrm{SN}$ rats at day 14. OVX: ovariectomy; SN: sciatic neurectomy; $E_{2}$ : 17ß-estradiol; CTRL: control. 
A

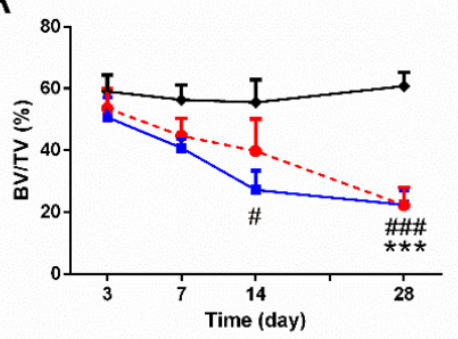

D

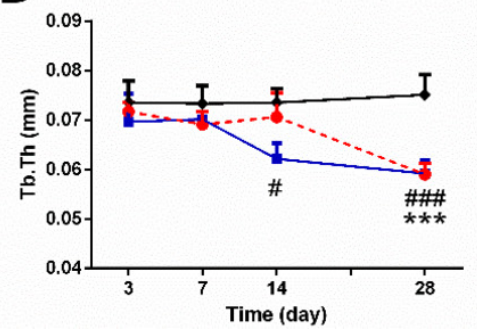

B

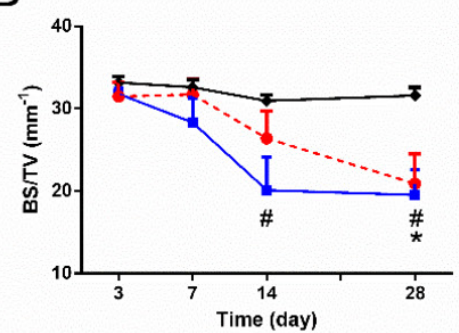

E

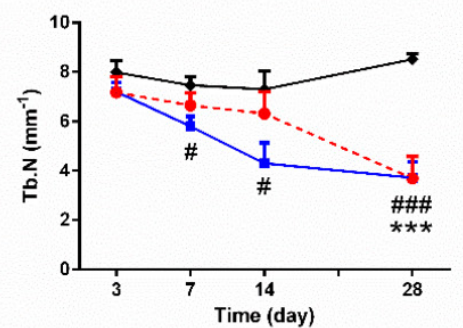

C

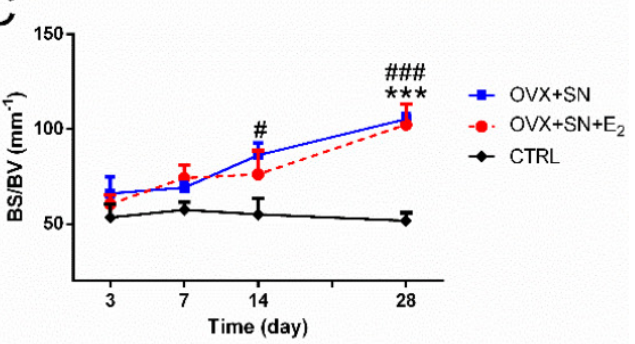

F

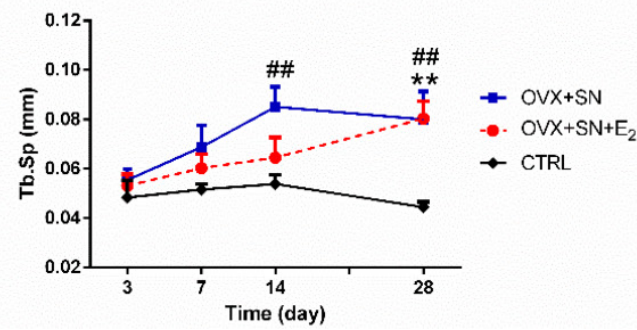

Fig. 2 Time-dependent changes in trabecular structural parameters assessed by micro-CT in the proximal tibia of OVX+SN rats treated with or without $E_{2}$, and CTRL rats. (A) BV/TV, bone volume fraction; (B) BS/TV, bone surface density; (C) BS/BV, bone surface/volume ratio; (D) Tb.Th, trabecular thickness; (E) Tb.N, trabecular number; and (F) Tb.Sp, trabecular separation. OVX: ovariectomy; SN: sciatic neurectomy; $E_{2}$ : 17ß-estradiol; CTRL: control. \# $\mathrm{OVX}+\mathrm{SN}$ vs. time-matched CTRL, $* \mathrm{OVX}+\mathrm{SN}+\mathrm{E}_{2}$ vs. time-matched CTRL. \# or $* \mathrm{P}<0.05$, \#\# or $* * \mathrm{P}<0.01, \# \#$ or $* * * \mathrm{P}<0.001$.

Fig. 2 shows the quantitative micro-CT analyses of BV/TV (Fig. 2A), BS/TV (Fig. 2B), BS/BV (Fig. 2C), Tb.Th (Fig. 2D), Tb.N (Fig. 2E), and Tb.Sp (Fig. 2F). There were no significant changes over time in these parameters in the CTRL rats. But dual surgeries of OVX and SN caused either significant decline in some parameters (BV/TV, BS/TV, Tb.Th, and Tb.N) or elevation in other (BS/BV and Tb.Sp), most starting at day 14 (day 7 for Tb.N). However, in all cases, $E_{2}$ treatment delayed the significant changes until day 28 after surgery.

The OVX+SN tibia receiving supplemental $E_{2}$ had less bone loss at day 14 post-surgery, as indicated by increased preservation of BV/TV and Tb.N, 43\% and $46 \%$ higher in $\mathrm{OVX}+\mathrm{SN}+\mathrm{E}_{2}$ rats than those in $\mathrm{OVX}+\mathrm{SN}$ rats, respectively (Fig. 2A, E). At day 28 after operation, however, these parameters almost reached similar levels in the $\mathrm{OVX}+\mathrm{SN}$ bone and $\mathrm{OVX}+\mathrm{SN}+\mathrm{E}_{2}$ bone.

The transient inhibition of bone loss by $E_{2}$ treatment was further confirmed by histological analysis (Fig. 3). The results showed that at day 14 post-surgery, BV/TV in the $\mathrm{OVX}+\mathrm{SN}$ group was significantly less than that in the control group. In contrast, $\mathrm{BV} / \mathrm{TV}$ in the $\mathrm{OVX}+\mathrm{SN}+\mathrm{E}_{2}$ rats was preserved by $58 \%$ compared with that in the $\mathrm{OVX}+\mathrm{SN}$ rats without $\mathrm{E}_{2}$ supplement at day 14 after surgery. However, the values of $\mathrm{BV} / \mathrm{TV}$ in both $\mathrm{OVX}+\mathrm{SN}+\mathrm{E}_{2}$ group and $\mathrm{OVX}+\mathrm{SN}$ group were identical at day 28 post-surgery, which were significantly decreased compared with the control group. Results from his- tological analysis were consistent with those from the micro-CT analysis.

\section{Serum changes of bone turnover markers}

Serum CTx level remained steady between days 3 and 28 in control rats and became significantly elevated between days 3 and 14 in OVX+SN rats, but returned to normal level at day 28 (Fig. 4A). In contrast, there was no significant difference in CTx level between $\mathrm{OVX}+\mathrm{SN}+\mathrm{E}_{2}$ and control rats (Fig. $4 \mathrm{~A}$ ).

Serum RANKL level did not change over time in control rats and was not impacted by surgeries between days 3 and 14, but, independent of $E_{2}$, rose significantly at day 28 compared with control rats (Fig. 4B).

\section{Sclerostin expression within osteocytes}

Our immunohistochemical assay for sclerostin demonstrated that sclerostin-positive osteocytes was expressed specifically in cortical bone, consistent with earlier observations [14]. The representative images of immunohistochemical staining for sclerostin showed that sclerostin-positive osteocytes (stained brown) were markedly increased over time in both $\mathrm{OVX}+\mathrm{SN}$ and $\mathrm{OVX}+\mathrm{SN}+\mathrm{E}_{2}$ bones compared with the corresponding control bone (Fig. 5A). A negative image confirmed the specificity of the sclerostin staining is shown as well (Fig. 5B). Quantitative analysis revealed that the percentage of sclerostin-positive osteocytes in $\mathrm{OVX}+\mathrm{SN}$ rats was elevated significantly at day 7 post-surgery relative to the corresponding con- 
trol rats, and remained high during the rest of the study (Fig. 5C). In contrast, the $\mathrm{OVX}+\mathrm{SN}$ rats with $\mathrm{E}_{2}$ treatment showed no significant increase in the percentage of sclerostin-positive osteocytes until 14 days post-surgery (Fig. 5C).

\section{Changes of osteoclasts}

The number of osteoclasts in the primary spongiosa remained steady over time in CTRL, but progressively declined from days 3 to 14 in OVX+SN (Fig. $6 \mathrm{~A}, \mathrm{~B})$. The decline in OVX+SN became significant at day 14, compared with CTRL, but fully recovered at day 28 (Fig.6A, B). With $\mathrm{E}_{2}$ treatment, although not significant, the number of osteoclasts trended higher than CTRL between days 7 and 14, back to normal at day 28 (Fig. 6A, B).

Between days 3 and 14 after operation, serum TRAP level in the OVX+SN rats was significantly reduced relative to that in the corresponding control rats, and then trended upwardly to day 28 (Fig. 6C), following the same trend as the number of TRAP-positive cells. With $\mathrm{E}_{2}$ treatment, serum TRAP 5 b level spiked at day 7 , then precipitated at day 14 , followed by a small recovery at day 28 compared with the corresponding control group (Fig. 6C).

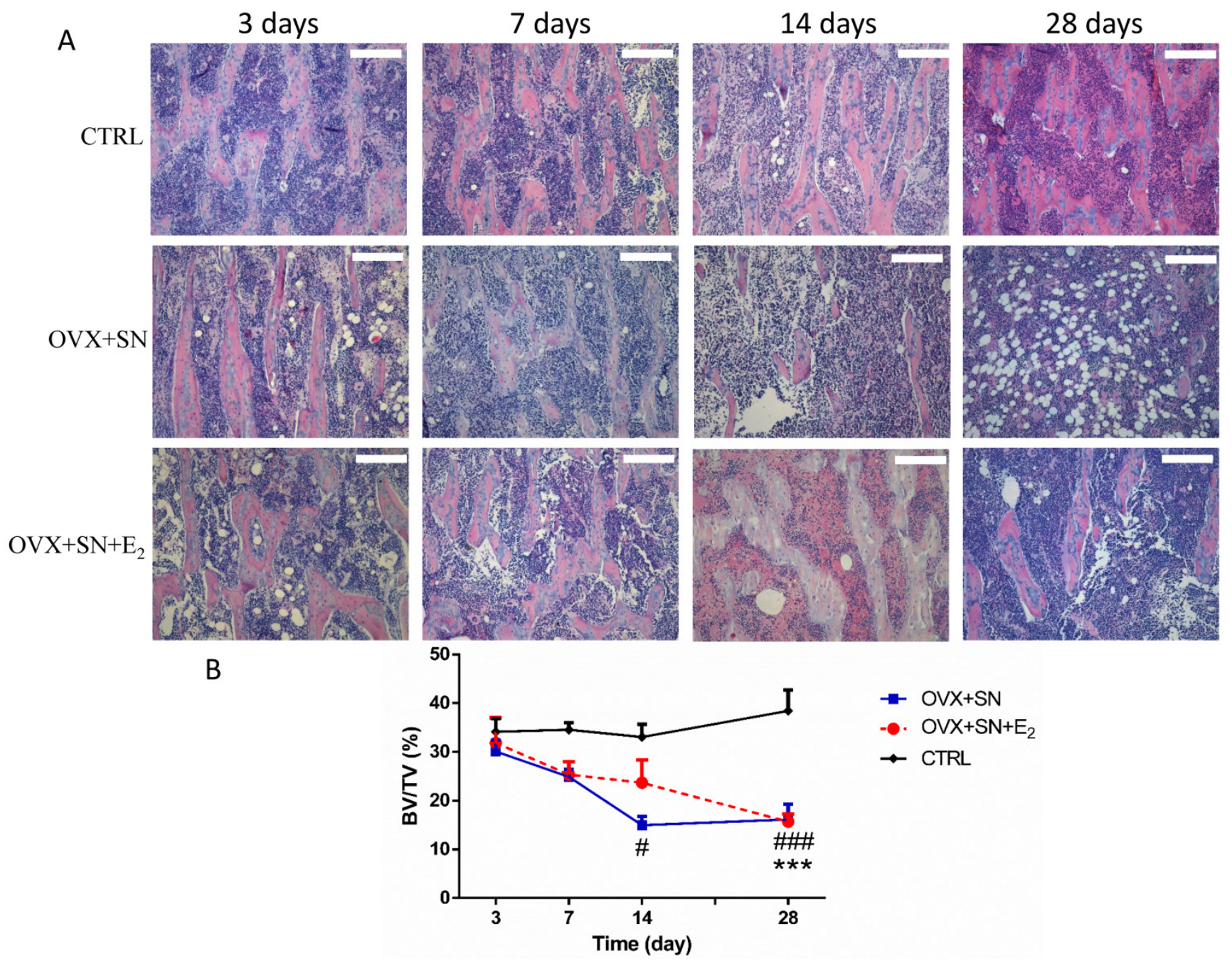

Fig. 3 Representative images $(10 x)$ and the ongoing changes on BV/TV by the histological analysis in the proximal tibia of OVX+SN rats treated with or without $E_{2}$, and CTRL rats. OVX: ovariectomy; $S N$ : sciatic neurectomy; $E_{2}: 17 \beta$-estradiol; CTRL: control. Bar $=200 \mu m$. \# OVX+SN vs. time-matched $C T R L$, * OVX+SN+E $E_{2}$ vs. time-matched CTRL. \# $P<0.05$, \#\# or $* * * P<0.001$ 
A

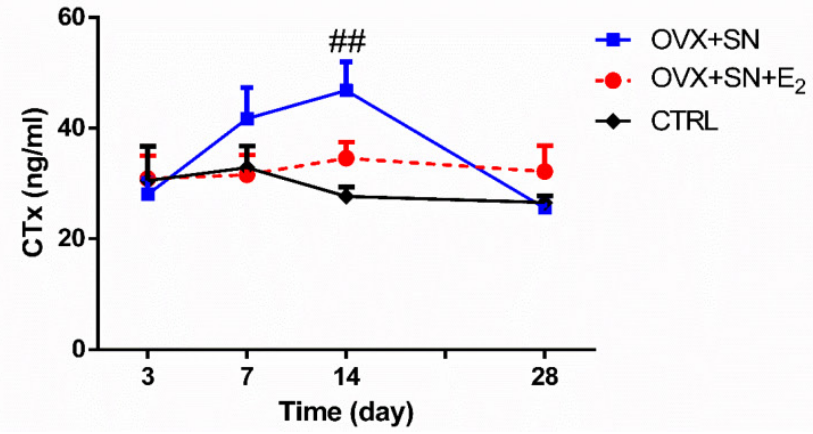

B

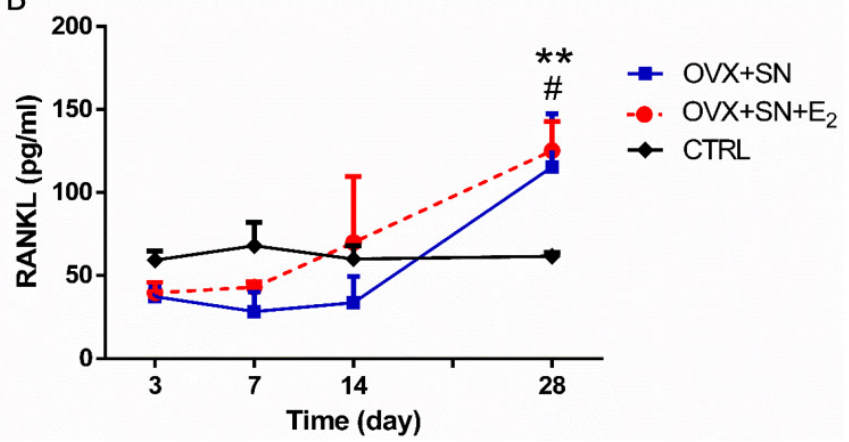

Fig. 4 The ongoing changes of serum CTx and RANKL in OVX+SN rats treated with or without $E_{2}$, and CTRL rats. (A) Serum CTx and (B) Serum RANKL. OVX: ovariectomy; SN: sciatic neurectomy; $E_{2}$ : 17ß-estradiol; CTRL: control. \# OVX+SN vs. time-matched CTRL, * OVX+SN+E2 vs. time-matched CTRL. $\#$ or $* \mathrm{P}<0.05, \#$ or $* * \mathrm{P}<0.01$, \#\# or $* * * \mathrm{P}<0.001$.

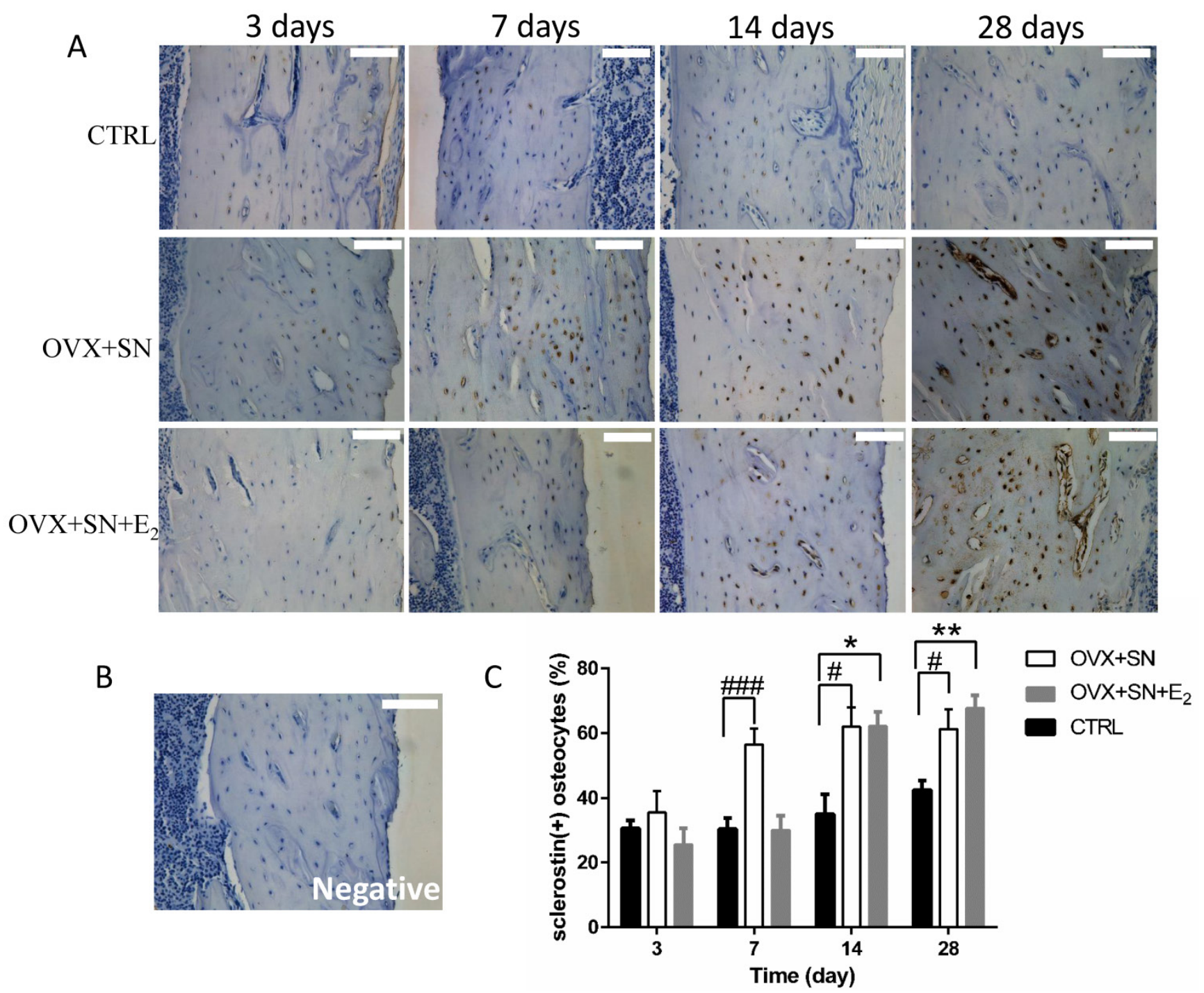

Fig. 5 The effect of OVX+SN with/without $E_{2}$ replacement on sclerostin expression in the proximal tibia. (A) Immunohistochemical images of sclerostin (20x, 3 days not shown). (B) A negative image confirming the specificity of the sclerostin staining is shown. (C) The ongoing changes of sclerostin-positive osteocytes in the proximal tibia of OVX+SN rats treated with or without $E_{2}$, and CTRL rats. OVX: ovariectomy; SN: sciatic neurectomy; $E_{2}$ : $17 \beta$-estradiol; $C T R L$ : control. Bar $=100 \mu \mathrm{m}$. \#OVX+SN vs. time-matched CTRL, * OVX+SN+E2 vs. time-matched CTRL. \# or $* \mathrm{P}<0.05$, \#\# or $* * \mathrm{P}<0.01$, \#\# or $* * * \mathrm{P}<0.001$. 
A CTRL

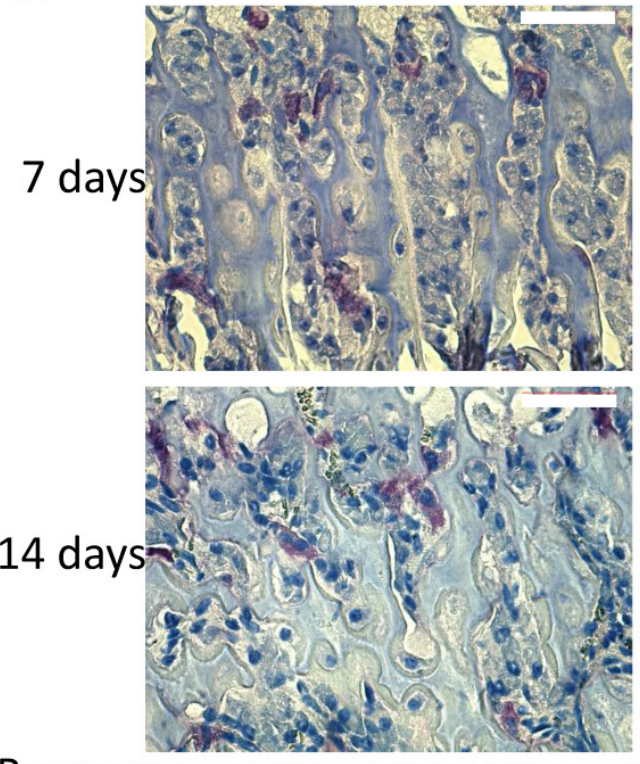

$\mathrm{B}$

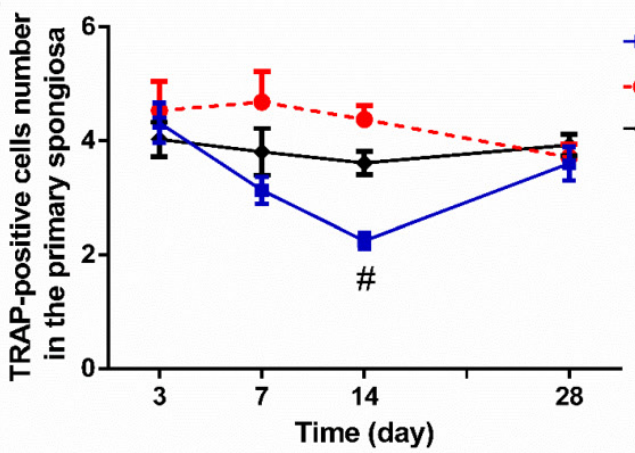

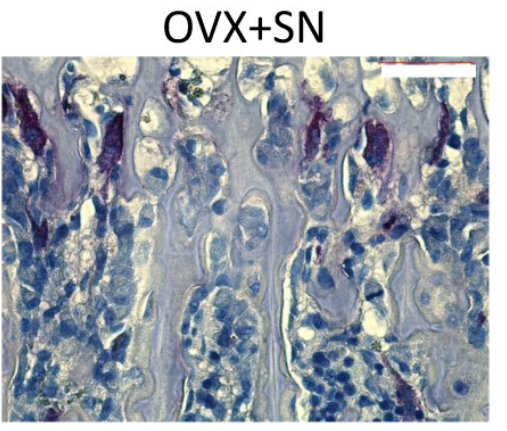
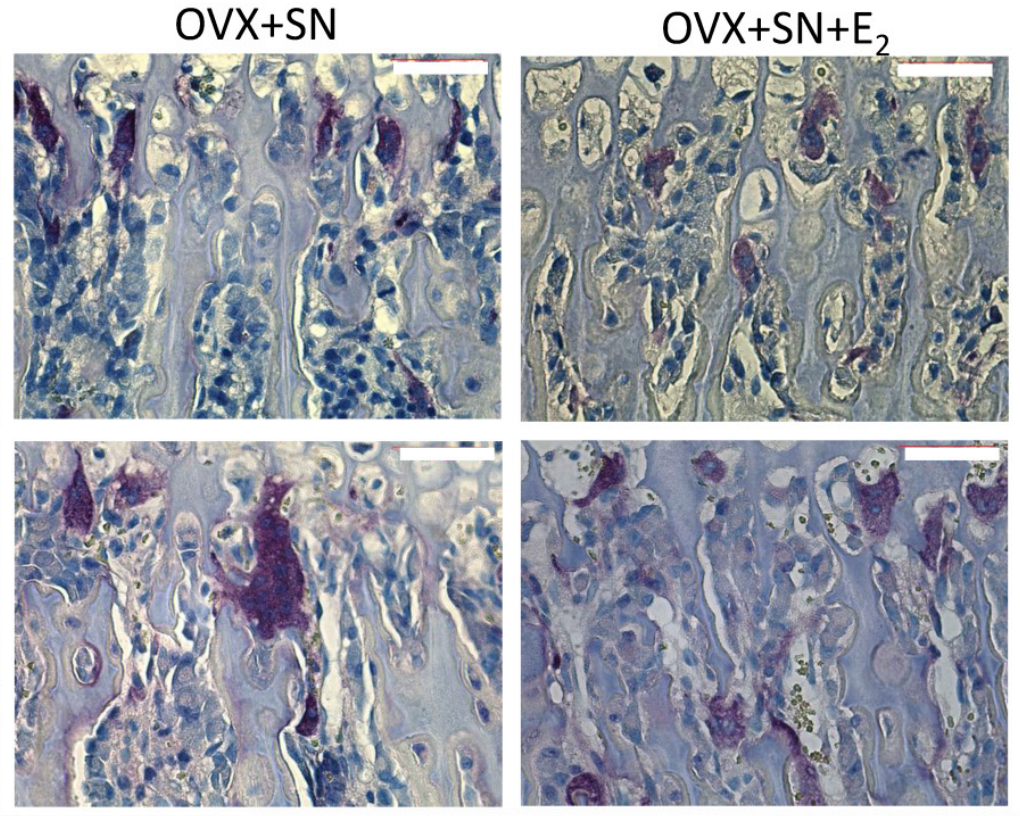

C

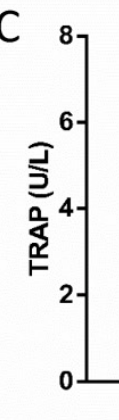

Fig. 6 Representative images and ongoing changes of TRAP $5 b$ in OVX+SN rats treated with or without $E_{2}$, and CTRL rats. (A) Representative images of TRAP $5 b$ staining in the primary spongiosa ( $40 \times ; 3$ and 28 days not shown). (B) The ongoing changes of the number of TRAP 5 b-positive cells in the primary spongiosa. (C) Serum TRAP 5b changes over a time course of 3, 7, 14 and 28 days. OVX: ovariectomy; SN: sciatic neurectomy; $E_{2}$ : $17 \beta$-estradiol; CTRL: control. Bar $=50 \mu \mathrm{m}$. \# OVX+SN vs. time-matched CTRL; * OVX+SN+E2 vs. time-matched CTRL. \# or $* \mathrm{P}<0.05$; \#\# or $* * \mathrm{P}<0.01$; \#\# or $* * * \mathrm{P}<0.001$.

\section{Discussion}

Estrogen influences bone development and maintenance, and estrogen replacement prevents bone loss resulting from estrogen deficiency in human [3]. However, the safety of estrogen treatment still remains a concern. There have been few studies evaluating the effect of estrogen replacement on bone loss in the postmenopausal women with mobility difficulties. Clinically, it is necessary to determine whether the benefits of bone preservation by estrogen treatment overweigh the potential risks of side effect. The current study using a rat model showed that $\mathrm{E}_{2}$ replacement reduced bone loss on the $\mathrm{OVX}+\mathrm{SN}$ tibia at 14 days post-surgery. However, quantitative micro-CT and histological data indicated that there was no significant difference in BV/TV and other parameters at day 28 post-surgery between the OVX+SN tibia and the $\mathrm{OVX}+\mathrm{SN}+\mathrm{E}_{2}$ tibia. This was kind of surprising considering the ability of $\mathrm{E}_{2}$ administration to prevent OVX-induced osteoporosis. One possible explanation is that either mechanical unloading or OVX or both impaired the estrogen receptor a expression within bone $[3,17]$, thereby diminishing the response of the bone to $\mathrm{E}_{2}$.

In our study, the selected markers, CTx and RANKL, showed an interesting time-dependent behavior under the conditions of estrogen-deficiency combined with unilateral sciatic neurectomy with/without $E_{2}$ therapy. The time-dependent changes in bone markers outlined their potential contributions to the phenotypic changes in the bone tissue, which provided us a deeper understanding on bone destruction. CTx, a degradation product of bone collagen, is a reliable marker of the resorption activity of osteoclasts [18]. RANKL is a vital factor of osteoclastogenesis and bone resorption [19]. In the current study, serum CTx levels increased gradually between days 3 and 14 in OVX+SN group, which was in agreement with the rapid bone destruction charac- 
terized by a steep slope of BV/TV. This might suggest that serum CTx level reflected either the extent of bone loss or the rate of bone destruction. However, serum CTx value reduced to normal level when the rate of bone deterioration became evidently slowed but bone volume remained little between days 14 and 28 days. This further implies that serum CTx levels reflect the rate of on-going bone deterioration rather than the volume of bone loss. In other word, when osteoporosis was very severe but the rate of bone loss was very slow, serum CTx level might not be elevated in patients with osteoporosis. Additionally, estrogen replacement inhibited CTx elevation to some extent. Serum RANKL levels increased significantly in the $\mathrm{OVX}+\mathrm{SN}$ rats and $\mathrm{OVX}+\mathrm{SN}+\mathrm{E}_{2}$ rats at 28 days after surgery but, not between days 3 and 14. The amplification of RANKL at 28 days post-surgery might partially explain the bone destruction in $\mathrm{OVX}+\mathrm{SN}$ bone and $\mathrm{OVX}+\mathrm{SN}+\mathrm{E}_{2}$ bone. However, we cannot explain the lack of increase of RANKL at day 14 in OVX+SN group while micro-CT data showed decreased bone mass at this time, although we realize that RANKL is a critical factor of bone resorption.

Sclerostin, the product of the SOST gene, is a negative regulator of bone formation and is secreted specially by the osteocytes [20]. Osteocytes have mechanosensory properties and can be triggered by mechanical loading to modulate bone homeostasis $[21,22]$. Modulation of sclerostin is influenced by the status of mechanical loading and/or estrogen [23, 24]. Mechanical loading down-regulates sclerostin expression within osteocytes [23, 25]. However, the effect of mechanical unloading on sclerostin/SOST expression varies. Robling et al.[23] found that hindlimb unloading yielded a significant increase in sclerostin expression in the tibia. Brandon et al. [20] showed the SOST mRNA expression in the cancellous metaphyseal bone was down-regulated at 3 days and 10 days after unloading and a similar trend was observed in the cortical bone at 10 days but, not at 3 days, after unloading. In the absence of estrogen, immunohistochemical results of sclerostin also vary: down-regulation [14], no significant change [26], and up-regulation [27]. However, it is the first study, to our knowledge, to explore the ongoing changes of sclerostin protein in ovariectomy model combined with sciatic neurectomy with/without estrogen replacement in the early phase. Our data showed that the combination of estrogen deficiency and disuse rapidly up-regulated the percentage of sclerostin-positive osteocytes at 7 days post-surgery and then maintained high levels to the end of experiment. However, with $E_{2}$ replacement, the up-regulated tendency of sclerostin expression was delayed, and until 14 days post-surgery, showed a significant in- crease. Estrogen treatment not only delayed sclerostin expression by osteocytes, but also transiently protected against trabecular bone loss. In agreement with the previous study, our immunohistochemical assay for sclerostin demonstrated that sclerostin-positive osteocytes was expressed specifically in cortical bone [14]. Sclerostin functions as a negative factor of bone formation, but it is still unclear how sclerostin is delivered by osteocytes, regulates the bone turnover, and its expression is inhibited by estrogen treatment.

TRAP $5 b$ is mainly produced by osteoclasts and is rich in osteoclast cytoplasm [28]. Serum TRAP 5b level reflects the number of osteoclasts rather than their activity [18, 29-33]. Previously, serum TRAP 5b values decreased at 2 weeks after OVX, because the histomorphometrically determined total number of osteoclasts in bone tissue is deceased due to substantial bone loss caused by the osteoporosis [18]. In our present study, serum TRAP $5 \mathrm{~b}$ levels were significantly lower in the OVX+SN group without $\mathrm{E}_{2}$ therapy at 14 days post-surgery than the control group, consistent with the number of TRAP-positive cells in the primary spongiosa. Additionally, both the number of TRAP-positive cells and the values of serum TRAP $5 \mathrm{~b}$ in the OVX+SN group trended back to normal at day 28, which may be correlated with the non-persistent bone loss as suggested by BV/TV values between days 14 and 28 and normal CTx levels at 28 days post-surgery. Although the number of TRAP-positive cells and serum TRAP $5 b$ in OVX+SN group showed a similar pattern of changes in this study, it should be noted that the number of TRAP-positive cells was measured from specific site, while the serum TRAP $5 b$ values describe the situation in the whole skeleton. This probably explains why the serum TRAP $5 b$ levels were not exactly consistent with the number of TRAP-positive cells in the primary spongiosa in the $\mathrm{OVX}+\mathrm{SN}+\mathrm{E}_{2}$ group. Especially considering that the relationship between the levels of bone loss and TRAP $5 b$, the results of serum TRAP levels and the number of TRAP-positive cells in the primary spongiosa supported the previous view that TRAP $5 \mathrm{~b}$ indicated the number of osteoclasts instead of their activity. In addition, $\mathrm{E}_{2}$ administration impacted dramatically the changes of serum TRAP $5 b$ in the OVX rats combined with mechanical unloading in the early phase. However, the long-term effects on the TRAP $5 \mathrm{~b}$ remain to be determined.

To our knowledge, the present study is the first to explore the ongoing changes of the combination of estrogen-deficiency and disuse with/without estrogen replacement in the early phase. However, it has a few limitations. The long-term and higher-dose $E_{2}$ treatments in this model remain to be determined, which must also be interpreted with caution due to 
the risk of estradiol. In addition, the expression of ERa is of great interest for future investigation which may help understand the underlying mechanism.

In summary, our results demonstrate that estrogen replacement can transiently protect against the bone loss in the estrogen deficiency combined with disuse. This finding may have potential clinical relevance given that our rodent model mimics the clinical condition under which estrogen-deficient women undergo disuse.

\section{Acknowledgement}

This work was funded by grant to $\mathrm{Y} \mathrm{Hu}$ from National Natural Science Foundation of China (Grant number: 81371124).

\section{Competing Interests}

\section{All authors declare no conflict of interest.}

\section{References}

1. Fujita K, Roforth MM, Demaray S, McGregor U, Kirmani S, McCready LK, et al. Effects of estrogen on bone mRNA levels of sclerostin and other genes relevant to bone metabolism in postmenopausal women. The Journal of clinical endocrinology and metabolism. 2014; 99: E81-8.

2. Brouwers JE, Lambers FM, van Rietbergen B, Ito K, Huiskes R. Comparison of bone loss induced by ovariectomy and neurectomy in rats analyzed by in vivo micro-CT. Journal of orthopaedic research : official publication of the Orthopaedic Research Society. 2009; 27: 1521-7.

3. Yarrow JF, McCoy SC, Ferreira JA, Pingel JE, Conrad BP, Wronski TJ, et al. A rehabilitation exercise program induces severe bone mineral deficits in estrogen-deficient rats after extended disuse. Menopause. 2012; 19: 1267-76.

4. Lee KC, Lanyon LE. Mechanical loading influences bone mass through estrogen receptor alpha. Exerc Sport Sci Rev. 2004; 32: 64-8.

5. Tou JC, Foley A, Yuan YV, Arnaud S, Wade CE, Brown M. The effect of ovariectomy combined with hindlimb unloading and reloading on the long bones of mature Sprague-Dawley rats. Menopause. 2008; 15: 494-502.

6. Kelley GA, Kelley KS. Exercise and bone mineral density at the femoral neck in postmenopausal women: a meta-analysis of controlled clinical trials with individual patient data. Am J Obstet Gynecol. 2006; 194: 760-7.

7. Kelley GA, Kelley KS, Tran ZV. Exercise and lumbar spine bone mineral density in postmenopausal women: a meta-analysis of individual patient data. J Gerontol A Biol Sci Med Sci. 2002; 57: M599-604.

8. Martyn-St James M, Carroll S. Meta-analysis of walking for preservation of bone mineral density in postmenopausal women. Bone. 2008; 43: 521-31.

9. $\mathrm{Ma} \mathrm{D}, \mathrm{Wu} \mathrm{L}, \mathrm{He} \mathrm{Z}$. Effects of walking on the preservation of bone mineral density in perimenopausal and postmenopausal women: a systematic review and meta-analysis. Menopause. 2013; 20: 1216-26.

10. Nikander R, Sievanen H, Heinonen A, Daly RM, Uusi-Rasi K, Kannus P. Targeted exercise against osteoporosis: A systematic review and meta-analysis for optimising bone strength throughout life. BMC Med. 2010; 8: 47.

11. Kohrt WM, Ehsani AA, Birge SJ, Jr. HRT preserves increases in bone mineral density and reductions in body fat after a supervised exercise program. J Appl Physiol (1985). 1998; 84: 1506-12.

12. Kohrt WM, Snead DB, Slatopolsky E, Birge SJ, Jr. Additive effects of weight-bearing exercise and estrogen on bone mineral density in older women. J Bone Miner Res. 1995; 10: 1303-11.

13. Galea GL, Meakin LB, Sugiyama T, Zebda N, Sunters A, Taipaleenmaki H, et al. Estrogen receptor alpha mediates proliferation of osteoblastic cells stimulated by estrogen and mechanical strain, but their acute down-regulation of the Wnt antagonist Sost is mediated by estrogen receptor beta. J Biol Chem. 2013; 288: 9035-48.

14. Jastrzebski S, Kalinowski J, Stolina M, Mirza F, Torreggiani E, Kalajzic I, et al. Changes in bone sclerostin levels in mice after ovariectomy vary independently of changes in serum sclerostin levels. J Bone Miner Res. 2013; 28: $618-26$

15. Iitsuka N, Hie M, Nakanishi A, Tsukamoto I. Ethanol increases osteoclastogenesis associated with the increased expression of RANK, PU.1 and MITF in vitro and in vivo. Int J Mol Med. 2012; 30: 165-72.

16. Hie M, Tsukamoto I. Administration of zinc inhibits osteoclastogenesis through the suppression of RANK expression in bone. Eur J Pharmacol. 2011; 668: 140-6.

17. Swift SN, Swift JM, Bloomfield SA. Mechanical loading increases detection of estrogen receptor-alpha in osteocytes and osteoblasts despite chronic energy restriction. J Appl Physiol (1985). 2014; 117: 1349-55.
18. Rissanen JP, Suominen MI, Peng Z, Halleen JM. Secreted tartrate-resistant acid phosphatase $5 \mathrm{~b}$ is a Marker of osteoclast number in human osteoclast cultures and the rat ovariectomy model. Calcif Tissue Int. 2008; 82: 108-15.

19. Kanematsu M, Sato $\mathrm{T}$, Takai $\mathrm{H}$, Watanabe $\mathrm{K}$, Ikeda $\mathrm{K}$, Yamada $\mathrm{Y}$. Prostaglandin E2 induces expression of receptor activator of nuclear factor-kappa B ligand/osteoprotegrin ligand on pre-B cells: implications for accelerated osteoclastogenesis in estrogen deficiency. J Bone Miner Res. 2000; 15: 1321-9.

20. Macias BR, Aspenberg P, Agholme F. Paradoxical Sost gene expression response to mechanical unloading in metaphyseal bone. Bone. 2013; 53: 515-9.

21. Suva LJ. Sclerostin and the unloading of bone. J Bone Miner Res. 2009; 24: 1649-50

22. Lin $C$, Jiang $X$, Dai Z, Guo X, Weng $T$, Wang J, et al. Sclerostin mediates bone response to mechanical unloading through antagonizing Wnt/beta-catenin signaling. J Bone Miner Res. 2009; 24: 1651-61.

23. Robling AG, Niziolek PJ, Baldridge LA, Condon KW, Allen MR, Alam I, et al. Mechanical stimulation of bone in vivo reduces osteocyte expression of Sost/sclerostin. J Biol Chem. 2008; 283: 5866-75.

24. Modder UI, Clowes JA, Hoey K, Peterson JM, McCready L, Oursler MJ, et al. Regulation of circulating sclerostin levels by sex steroids in women and in men. J Bone Miner Res. 2011; 26: 27-34.

25. Galea GL, Sunters A, Meakin LB, Zaman G, Sugiyama T, Lanyon LE, et al. Sost down-regulation by mechanical strain in human osteoblastic cells involves PGE2 signaling via EP4. FEBS Lett. 2011; 585: 2450-4.

26. Miyagawa K, Kozai Y, Ito Y, Furuhama T, Naruse K, Nonaka K, et al. A novel underuse model shows that inactivity but not ovariectomy determines the deteriorated material properties and geometry of cortical bone in the tibia of adult rats. Journal of bone and mineral metabolism. 2011; 29: 422-36.

27. Jia HB, Ma JX, Ma XL, Yu JT, Feng R, Xu LY, et al. Estrogen alone or in combination with parathyroid hormone can decrease vertebral MEF2 and sclerostin expression and increase vertebral bone mass in ovariectomized rats. Osteoporosis international : a journal established as result of cooperation between the European Foundation for Osteoporosis and the National Osteoporosis Foundation of the USA. 2014; 25: 2743-54.

28. Janckila AJ, Parthasarathy RN, Parthasarathy LK, Seelan RS, Hsueh YC, Rissanen J, et al. Properties and expression of human tartrate-resistant acid phosphatase isoform 5 a by monocyte-derived cells. J Leukoc Biol. 2005; 77: 209-18.

29. Alatalo SL, Halleen JM, Hentunen TA, Monkkonen J, Vaananen HK. Rapid screening method for osteoclast differentiation in vitro that measures tartrate-resistant acid phosphatase $5 \mathrm{~b}$ activity secreted into the culture medium Clin Chem. 2000; 46: 1751-4.

30. Alatalo SL, Peng Z, Janckila AJ, Kaija H, Vihko P, Vaananen HK, et al. A novel immunoassay for the determination of tartrate-resistant acid phosphatase $5 \mathrm{~b}$ from rat serum. J Bone Miner Res. 2003; 18: 134-9.

31. Chu P, Chao TY, Lin YF, Janckila AJ, Yam LT. Correlation between histomorphometric parameters of bone resorption and serum type $5 \mathrm{~b}$ tartrate-resistant acid phosphatase in uremic patients on maintenance hemodialysis. Am J Kidney Dis. 2003; 41: 1052-9.

32. Alatalo SL, Ivaska KK, Waguespack SG, Econs MJ, Vaananen HK, Halleen JM. Osteoclast-derived serum tartrate-resistant acid phosphatase $5 \mathrm{~b}$ in Albers-Schonberg disease (type II autosomal dominant osteopetrosis). Clin Chem. 2004; 50: 883-90.

33. Li X, Ominsky MS, Warmington KS, Niu QT, Asuncion FJ, Barrero M, et al. Increased bone formation and bone mass induced by sclerostin antibody is not affected by pretreatment or cotreatment with alendronate in osteopenic, ovariectomized rats. Endocrinology. 2011; 152: 3312-22. 